\title{
Abdominal injuries
}

\author{
C. J. ANDERS \\ F.R.C.S.
}

Consultant Surgeon, St Peter's Hospital, Chertsey

\begin{abstract}
AbDominal injuries pose a difficult problem for the Accident Centre or Casualty Department. Patients may present with other injuries which are more obvious or more important, and it is easy to overlook an abdominal condition. It must be stressed that a full examination is essential in all cases, no matter how trivial the injury may have been. It is difficult to separate the question of abdominal injuries from the consideration of the patient as a whole, but if one is to try and do so, then they come high on the list of priorities when dealing with a casualty. In some accidents the injuries are so severe that death occurs before arrival at the hospital. These are usually severe head injuries or thoracic injuries, but may also be due to intra-abdominal damage. If the patient survives, abdominal injuries present clinically in one of two ways, either as the result of haemorrhage and blood loss, or else as peritonitis following spillage of the contents of a viscus. All haemorrhage requires treatment. If external it is obvious enough. Intra-abdominal haemorrhage must be treated with equal promptness, and is all the more dangerous because it is hidden.
\end{abstract}

\section{Sites of injury}

In considering abdominal injuries, it is as well to remember the different structures that may be injured. The following classification is suggested as a help in making a diagnosis.

(1) Abdominal wall injuries. Skin, subcutaneous tissues, and muscle.

(2) Extraperitoneal injuries. Retroperitoneal haemorrhage, tears of the aorta or inferior vena cava, fractured ribs, pelvis or vertebrae.

(3) Intraperitoneal injuries. Liver, spleen, stomach, small bowel, colon, mesentery and pancreas.

(4) Genito-urinary tract injuries. Kidney, ureter, bladder and urethra.

\section{Causes}

Abdominal injuries may result from a wide variety of causes. These can be considered under three main headings:

(1) Blunt or non-penetrating injuries.

(2) Penetrating injuries.

(3) Compressed air injuries.
Much of what is said under the first of these headings will also apply to the other types, which are discussed more briefly.

\section{Blunt injuries}

Blunt or non-penetrating injuries of the abdomen are due to a blow such as a kick, punch, or sudden impinging of any hard body against the abdominal wall. The commonest of all such injuries seen today follow automobile accidents. Compression injuries are included in this category, and here it is the driver of the vehicle who is affected in particular. He suffers the so-called 'steering wheel injury' as he is thrown forwards and impaled by an unyielding steering column. This results in fractured ribs and/or damage to any of the intra-abdominal organs, but the liver is the most vulnerable. Bowel may be compressed between the steering wheel and the vertebrae producing tears of the mesentery or ruptures. of the bowel. A more severe crushing injury would follow from a heavy vehicle passing over the abdomen. A fall from a height will produce excessive strain on the visceral attachments, and can result in tears.

\section{History}

The history is always of some value, and the site of the injury should be noted as well as its severity. The story is not always reliable, however, and it is often necessary to deal with an unconscious patient. In those cases where violence has been applied to the abdominal wall, the kind of injury produced will depend to some extent on the preparedness of the patient. If he has been able to tighten his abdominal muscles, the injury is likely to be less severe. In some instances, the patient may not survive long enough to even reach the hospital. At the other extreme, the cause is quite trivial, such as the case of the young father playing with his 2-year-old son who kicked him in the abdomen, and the result was a ruptured spleen.

It is important to ascertain whether the patient has been drinking, and whether he has recently eaten anything. If inebriated the physical signs will be difficult to elicit and interpret. Such patients may have a full bladder at the time of injury, and the possibility of a ruptured bladder must not be forgotten. 
Haematemesis suggests that the patient has either swallowed blood from the naso-pharynx, or has sustained damage to the stomach. Bleeding per rectum suggests trauma to the lower gastro-intestinal tract.

Pain on the tip of the shoulder may be a referred pain as a result of irritation of the diaphragm.

\section{Physical examination}

The diagnosis of abdominal injury depends very largely on physical examination. It is important to remember the two main effects of trauma, which are haemorrhage and spillage of the contents of the hollow viscera. Either may occur alone, or there may be a combination of the two.

The signs of haemorrhage are too well known to require detailed elaboration. The patient will become pale and sweating occurs. The pulse rate will rise, and become weak and thready. The blood pressure will fall. If these signs develop, and there is no external haemorrhage, and no collection of fluid in the pleural cavity, then it must be assumed that there has been an intra-abdominal haemorrhage until proved otherwise. Blood in the peritoneal cavity will result in peritoneal irritation and will give rise to the signs of peritonitis. Spillage of intestinal content will also give rise to peritonitis.

The detailed examination of the abdomen is the same as for any other abdominal condition.

Inspection. This may reveal bruising of the skin, distension of the abdomen, or poor movement on respiration.

Palpation. This is without doubt the most valuable examination of all in reaching a diagnosis following an injury, or indeed for any other abdominal condition. However, it is extremely difficult to describe adequately what one should look for on palpation, and it is only with experience that it is possible to interpret correctly the sensations obtained by the examining hand. Light palpation should be done first with a minimum of pressure, using only the tips of the fingers, to determine any tenderness, guarding or rigidity. When the whole abdomen has been thoroughly examined in this way, deep palpation may then be done, slowly and gently, leaving till last the area of maximum tenderness. Each patient reacts in a different way, and it is important to watch closely the face of the patient to see his reaction whilst this is being done. It should be possible to say whether there is peritonitis present in a large percentage of patients. It must be remembered that bruising of the abdominal muscles results in tenderness and guarding without any intraabdominal pathology.

Testing for rebound tenderness is advised by some, but the author feels strongly that it never gives any additional information to that already obtained by careful palpation. It merely hurts and results in loss of confidence by the patient.
The finding of a mass will be noted, and it is a useful trick to outline the edge of the swelling with a skin marker, so that it can be easily compared later to see if there has been any increase in size. The presence of a swelling is likely to be due to a haematoma, and rapid increase in size means continued haemorrhage.

Auscultation. The presence of bowel sounds is an extremely good indication that there is no serious underlying trouble.

Some authors writing on this subject feel that if bowel sounds are present following a blunt injury, laparotomy is not necessary. They state that if they are absent, laparotomy should always be undertaken as this means peritonitis has developed, either as a result of blood or spillage from a viscus. It should be noted that a retro-peritoneal haematoma may cause a reflex ileus and absence of bowel sounds does not, therefore, always necessarily mean peritonitis.

Percussion. Free fluid will be shown by the demonstration of shifting dullness. After an injury, this suggests blood in the peritoneal cavity. It requires quite a large haemorrhage to produce enough fluid and the absence of shifting dullness does not rule it out. Percussion of the loins is particularly useful here, because if, in addition to the shifting dullness, there is a persistent dullness in one or other loin, this represents a retroperitoneal haematoma on that side.

\section{Special investigations}

Careful clinical examination is the most important assessment of an abdominal injury, but other investigations may give valuable and useful information.

Haemoglobin and PCV. In any acute haemorrhage these values may remain unaltered in the initial phases until haemodilution has taken place. However, these should be evaluated in all cases.

White cell count. There is a tendency to a leucocytosis following injury, but it is unpredictable and is not a reliable test.

Cross-matching. All patients with suspected haemorrhage need to be grouped, and serum retained for the cross-matching of blood as required.

Urine examination. The patient should be asked to pass urine and this is then examined to determine the presence of blood. Microscopic amounts are probably not of great significance immediately following an injury. Macroscopic haematuria means damage to either kidney or bladder, and less often to the prostate or urethra. Catheterization should be avoided in the Accident Centre.

$X$-ray. This may give valuable information, but it cannot be stressed too strongly that immediate treatment for blood loss and arrest of haemorrhage is far more important than obtaining an X-ray. 
Obvious and continued haemorrhage is an indication that emergency laparotomy should be done. It is possible for a patient to bleed to death whilst waiting for an X-ray that would have given no help.

In the doubtful case a straight X-ray may be helpful. Air under the diaphragm indicates a ruptured viscus. Fractured pelvis and/or ribs suggest the possibility of damage to certain intraabdominal organs such as urethra, bladder, spleen, liver or diaphragm. Fractures of the pelvis are often associated with massive haemorrhage, and several pints of blood may be lost in the tissues.

If rupture of the spleen is suspected, the following signs should be looked for on the plain X-ray: enlargement of the splentic shadow, a raised diaphragm on the left side, fracture of one or more of the lower ribs on the left side, loss of outline of the of the left kidney or psoas shadow, gastric dilatation, local indentation or irregularity of the stomach, medial displacement of the fundus of the stomach, presence of free fluid in the peritoneal cavity as evidenced by a 'ground-glass' appearance, and downward displacement of the splenic flexure. One or more of the above features is suggestive of damage to the spleen, but it must be remembered that the $X$-ray is normal in $15-20 \%$ of cases involving a tear of the spleen.

IVP. An emergency intravenous pyelogram may be of value in demonstrating function of the kidney. A simple technique of injecting contrast media intravenously, followed by a single exposure 15-20 min later will give valuable information and it is not necessary to produce a full series of pictures as in the normal IVP examination. Two things can be demonstrated with a single picture; first, if there is function in both kidneys, and secondly, if all or part of the calyceal system is outlined. No function on one side means either complete absence of the kidney or else severe damage. In this emergency technique, concentration of the dye is poor and tomograms give additional information. It must be remembered that forward displacement of the kidney may have occurred following a retroperitoneal haematoma and, therefore, if only one kidney is demonstrated several cuts must be made if tomograms are to be accurately interpreted.

Peritoneal tap. This procedure is not widely used in this country, but some American authors go so far as to consider it the most valuable of all investigations. It is usually possible to arrive at a reasonably accurate diagnosis in most cases without this test, but nevertheless it is worth consideration. The technique is very easy, and simply consists of the insertion of a needle with syringe attached, into all four quadrants of the abdomen. There is a characteristic sensation and a 'give' as the needle enters the peritoneal cavity. If done slowly with the needle at right angles to the surface, there is virtually no danger at all to the bowel. Aspiration is then carried out. The presence of blood is diagnostic of haemorrhage. Free fluid or purulent material is likely to be associated with rupture of a viscus. A modification of this technique is to use a large-bore needle through which a ureteric catheter can be passed. If the needle is then angled and the catheter threaded through the needle, it can be directed towards the posterior abdominal wall and the paracolic gutters, thus obtaining a more representative sample.

This is an easy procedure and it is perhaps most useful in the unconscious patient, particularly in those with head injuries or shock due to other causes. It can also be of value in the doubtful case where diagnosis is not certain. A positive tap is of great help, but a negative tap must never be interpreted as meaning that there is no trouble in the peritoneal cavity.

\section{Blood volume estimation}

If the hospital is equipped with a Volumetron, this instrument may be used to estimate blood volume and, therefore, blood loss. The test is really a dilution test, using radioactive iodine. A measured dose is given, taking samples of blood immediately beforehand and again after a period of $10 \mathrm{~min}$ when it is assumed that adequate mixing will have taken place. The dose to be given and the two samples of blood are then inserted into the machine which contains a Geiger counter and a small computer which calculates the result automatically. The figure obtained is then read off against a chart which takes into account the patient's height and weight. On theoretical grounds this estimation should be the most accurate of all in determining blood loss, but it has been found that in practice the figures obtained are not always reliable, and care must be taken in the interpretation of the results.

\section{Laparotomy}

If intra-abdominal damage has been diagnosed, laparotomy must be undertaken with two objects in mind; to stop and control haemorrhage, and to repair any defect. In cases of a doubtful nature the patient should be kept under observation, preferably in an Accident Centre, or detained in a hospital ward. Only careful and continued observation in these patients will determine whether operation should be done or not. Despite this, a certain proportion of patients are likely to undergo a laparotomy at which nothing is found. This is probably inevitable, and in most articles written on this subject it is admitted that it is very difficult to avoid this. Routine operation carries a definite mortality and morbidity, but on the other hand it is wiser to perform a needless laparotomy than to miss the opportunity to repair a remedial defect. 


\section{Discussion}

A precise diagnosis in abdominal injury is not important, and it is sufficient to diagnose either haemorrhage or peritonitis. Injuries are frequently multiple, but it is worth while considering some of the features that may be associated with different sites of injury. If it is possible to pinpoint the damage, this may influence the management of the case.

(1) Contusion to the abdominal wall is suggested by the history, local tenderness, visible swelling and bruising. It is, of course, important to differentiate this type of injury as surgery is practically never indicated.

(2) Retro-peritoneal haemorrhage which develops as a result of damage to small vessels, muscular tears, or is secondary to fractures of bones, seldom requires surgical intervention. It is a different matter if major vessels such as the aorta or inferior vena cava are torn, and in these cases the amount of haemorrhage is likely to be copious.

(3) Splenic damage. A history of a blow in the upper left quadrant or over the left lower ribs followed by a rise in pulse, pallor and a fall in blood pressure is almost certainly due to a tear of the spleen. X-ray and peritoneal tap are useful aids in diagnosis. Delayed splenic rupture is not uncommon and occurs between the 7th and 14th days, though cases have been reported where it did not occur until 3 months later. Ruptured spleen is an emergency and haemorrhage may be large.

(4) Gastro-intestinal tract. Injuries involving stomach, small bowel and colon may result in haemorrhage and/or rupture. Tears have a tendency to occur near to fixed parts, for example the first few inches of the jejunum which is adjacent to the ligament of Treitz. The clinical assessment of peritonitis is of most value in reaching a diagnosis. Erect X-ray which reveals gas under the diaphragm means a ruptured viscus.

(5) Liver or biliary tract. Here there is a history of a blow over the liver which is followed by the clinical picture of shock and the signs of peritoneal irritation. The commonest site of injury is in the dome of the right lobe of the liver. Loss of blood may be great, and like the spleen, liver injuries should be treated as emergencies.

(6) Pancreatic injuries. There are no special features, but shock may be severe. There may be a rise in serum amylase.

(7) Kidney. Minor damage and bruising of the kidney is common and can only be inferred by the presence of haematuria. IVP is often entirely normal. As stated above, tomograms may give additional information.

\section{Priority of treatment}

Details of how to deal with a casualty are not considered here, but it is worth emphasizing the basic essentials of emergency treatment in the accident centre or casualty department.

(1) Restore and maintain airway.

(2) Restore normal pulmonary function.

(3) Control haemorrhage.

(4) Restore blood loss.

\section{Emergency action for abdominal injuries}

In abdominal injuries the most urgent condition to be faced is internal haemorrhage, and this is an emergency. The same principles apply here as for any other patient who has lost blood. A sample of blood must be obtained for subsequent grouping and cross-matching. An intravenous drip must be set up and treatment for shock instituted. Blood volume must be restored as soon as possible, ideally by blood where it is available. Plasma or a plasma substitute such as Dextran will provide temporary resuscitation until blood is available. If there is evidence of continued haemorrhage, laparotomy must be carried out as soon as the patient has been rendered fit for operation. If there are fractures of long bones, these should be stabilized with temporary external splints, but fixation must be deferred until all haemorrhage has been stopped.

Laparotomy is best done through a vertical incision, either a paramedian or midline, which is quick and easy. Bleeding frequently increases as the abdomen is opened. A useful trick for arterial haemorrhage is to compress the aorta just below the diaphragm as a temporary measure, but this will not control venous bleeding.

Peritonitis which follows a ruptured viscus is not, perhaps, quite so urgent. All these patients will develop a paralytic ileus. The treatment is the same as for peritonitis from any other cause. Intravenous fluids must be given and a nasogastric tube passed to decompress the stomach. Blood must be taken for grouping and cross-matching. Physiological saline $(0.9 \%)$ is the fluid of choice when setting up the intravenous drip. Blood, plasma or its substitutes are not indicated unless there is evidence of associated haemorrhage. Laparotomy should be done as soon as convenient, and repair or resection of the damaged viscus carried out as indicated.

Specific intra-abdominal injuries will only be discussed briefly with regard to their treatment. Splenic injury requires splenectomy. Small perforations of stomach or small intestine may be treated by simple closure. Larger perforations and tears of the mesentery require resection and end-to-end anastomosis. Large bowel injuries may require colostomy. Renal injuries, if slight, require no treatment and even severe injuries make an excellent 
recovery on conservative management. Continued bleeding may require partial or complete nephrectomy. Diaphragmatic tears should be sutured, and this can be done easily in most cases. Pancreatic injuries are more difficult, and it may be necessary to amputate the body and tail in some. Liver injuries are notoriously difficult. Devitalized tissue is to be removed. Tears may be sutured; Gelfoam or Oxycel is sometimes helpful. Occasionally insertion of a large pack may be the only way to control the bleeding. Injuries to the liver and pancreas must always be drained.

\section{Penetrating wounds of the abdomen}

Commoner in wartime or in military service, these injuries are fortunately seen less often in peacetime. Penetrating wounds are produced by a variety of methods. The most frequent in war are wounds by bullets or explosion. In civilian practice the incidence of stabbing is on the increase.

There is a fundamental difference between blunt injuries and penetrating wounds. In the latter the decision is no longer whether an operation should be done, but rather when it must be done. Infection is frequently introduced as a complication.

History. The history is of importance in discovering the position or attitude of the patient. Entry and exit wounds must both be looked for. A basic assumption is that the missile will pass in a straight line. Lesions in widely separated portions of the small bowel are due to two factors: changes in the position of the viscera and the attitude of the patient's body at the time of injury. The necessity for locating any missile between the shoulder and the knee cannot be stressed too strongly.

Physical examination. The main signs of peritoneal irritation are reliable, and are similar to those discussed above when dealing with blunt injuries. The patient will complain of pain. Palpation again yields the most valuable information of all when examining the patient. Tenderness, guarding and rigidity must be looked for and carefully assessed. Bowel sounds that are decreased or absent are very suggestive of peritonitis. X-ray examination is of great value in locating the missile, and must always be done in two planes.

\section{Stab wounds}

History. An accurate history is often difficult to elicit. Many patients are confused from alcohol and blood loss. Some are afraid of police action or of further attacks. Witnesses are sometimes able to provide useful information, but they too may be reticent for the same reasons. The length of the knife blade should be ascertained wherever possible.
Physical examination. A note should be made of the clothing that is worn. The position of the stab holes will help in finding the direction of the injury. Thick winter clothing may have absorbed much of the blow. An attempt should be made to estimate the amount of blood lost. As with any other injury, the patient's general condition, pulse, and blood pressure must all be recorded.

The site and size of the wounds must be determined with particular reference to the back. It is easy, when faced with a piece of omentum protruding from an abdominal wound, to treat the obvious wound and forget to inspect the back. Such a wound may, in fact, be an exit wound from a stab in the back. Stab wounds are frequently multiple. Intra-abdominal wounds may also result from stabs in the thorax or buttock.

Stab wounds involving only the abdominal wall cause tenderness and guarding in the region of the injury. If the wound is only superficial, these signs will regress, tut in the management of this type of injury this is really of academic interest. All wounds must be explored to assess direction, depth and damage to internal organs. There is no place for conservative treatment following a stabbing.

\section{Emergency action for penetrating wounds}

The same rules apply as for blunt injuries thas have been described earlier. In addition, all thes. patients must have their wounds thoroughly explored, preferably under general anaesthetic. As before, an intravenous drip should be set up, blood taken for grouping and cross-matching, and a nasogastric tube passed. Infection is very likely to have been introduced and routine antibiotics are a wise precaution. Wounds should be covered with sterile towels. If bowel or omentum is protruding, no effort should be made to reduce this until the time of laparotomy.

\section{Compressed air injuries}

These are relatively uncommon. The history is clear in that a compressed air apparatus has been applied to the anal orifice, usually an unfortunate junior apprentice at a factory or garage, whilst someone with a perverted sense of amusement turns on the pressure. If this is too great, rupture of the rectum or sigmoid will result, and both air and faeces will escape into the peritoneal cavity. There are no special features apart from the history, and clinical examination reveals the signs of peritonitis. X-ray shows free gas under the diaphragm in a proportion of cases. All cases must be treated surgically, and a colostomy may be required. 